\title{
Enhancement of Renal Tubular Epithelial Cell Barrier Function by the Immunosuppressant Drugs Cyclosporine A and Sirolimus
}

\author{
Craig Slattery, Natalia Martin-Martin, Tara McMorrow and Michael P. Ryan*
}

UCD School of Biomolecular and Biomedical Sciences, UCD Conway Institute, University College Dublin, Belfield, Dublin 4, Ireland

\begin{abstract}
The primary function of the kidney includes the elimination of waste products and the maintenance of fluid and electrolyte composition of the body. The functioning unit of the kidney is the nephron including the glomerulus with a filtration function and the tubule with a reabsorption and secretion function. The fluid and electrolyte homeostasis is achieved largely through selective, vectorial transport facilitated by the renal tubular epithelium. This involves both transcellular and paracellular transport. Paracellular transport across renal tubular epithelial tight junctions (TJs) varies in different parts of the nephron. The relative concentrations of different claudins that are located in the TJ at any time determine the ion and size selectivity of the paracellular diffusion pathway in the kidney as in other tissues. In this article, we review work from our own laboratory and others providing evidence that immunosuppressive drugs, especially cyclosporine A (CsA) and sirolimus (SRL) and can alter transport molecules in kidney tubules. We outline evidence that CsA and SRL can enhance renal epithelial barrier function as indicated by transepithelial electrical resistance (TEER). The possible signalling mechanisms involved in altering the TJs and claudins by CsA and SRL are outlined and involve both TGF- $\beta 1$ and the ERK 1/2. These effects of CsA and SRL on kidney epithelia cell barrier function may help to explain some of the renal transport defects and nephrotoxicity seen with CsA and SRL. However if similar effects occur systemically in vivo, with these two immunosuppressive drugs, the enhancement of barrier function in areas of the body may decrease luminal antigen presentation, decreasing immune activation and support the immunosuppressive action of these drugs.
\end{abstract}

Keywords: Tubular epithelial cells, rapamycin, sirolimus, cyclosporine.

\section{BARRIER FUNCTION OF THE RENAL TUBULAR EPITHELIUM}

The primary function of the kidney includes the elimination of waste products and the maintenance of fluid and electrolyte composition of the body. The functioning unit of the kidney is the nephron including the glomerulus with a filtration function and the tubule with a reabsorption and secretion function. The fluid and electrolyte homeostasis is achieved largely through selective, vectorial transport facilitated by the renal tubular epithelium. The reabsorption and secretion of various solutes across the renal tubular epithelium constitutes a major aspect of kidney function. The primary characteristic of all epithelial cells, including kidney epithelial cells, is the ability to act as selective barriers between compartments in the body. This is accomplished by the fact that epithelial cells are polarized (i.e. they have distinct apical and basolateral domains). Therefore, epithelial layers can act as asymmetric transporters of salts, nonelectrolytes and water thereby regulating the volume and composition of the compartments on either side. Epithelial cells adhere to each other through molecular complexes that form junctions between the cells. These intercellular junctions not only fulfil a structural, adhesive function but also

\footnotetext{
*Address correspondence to this author at the UCD Conway Institute for Biomolecular and Biomedical Research, University College Dublin, Belfield, Dublin 4. Ireland; Tel: +353-1-7166819; Fax: +353-1-7166456;

E-mail: michael.p.ryan@ucd.ie
}

exert significant influence over epithelial cell proliferation, differentiation, and over the barrier function of the layer $[1,2]$.

There are two routes by which ions may traverse the renal tubular epithelium - transcellular and paracellular transport. The regulation and maintenance of transcellular and paracellular transport is vital for the normal function of many organs, including the kidney. These routes facilitate movement of solutes, water and essential ions such as sodium, chloride, calcium and magnesium across epithelia and are critical for the maintenance of electrolyte balance and total body fluid and electrolyte homeostasis. Transcellular transport is mainly catalyzed by specific membrane pumps and channels on the apical and basolateral membrane. These transport processes generate transepithelial electrochemical and osmotic gradients which then drive the passive movement of solutes through the paracellular pathway, which is size and charge selective for small molecules and electrolytes $[1,2]$. The 'tightness', or conversely, the 'leakiness' of an epithelial barrier layer is largely determined by the intercellular junctions including the adherens junctions, desmosomes and especially the tight junctions (also known as the zonula occludens). The adherens junctions and desmosomes act largely as structural adhesive junctions and are anchored to the actin cytoskeleton and intermediate filaments respectively. 


\section{RENAL EPITHELIAL TIGHT JUNCTIONS}

The tight junctions (TJs) however, are the most apical junctional structure encircling the cell at the apical portion of the lateral membrane and forming the primary paracellular diffusion barrier which regulates epithelial permeability $[2$, 3]. Paracellular transport across renal tubular epithelial TJs varies in different parts of the nephron. The TJ barrier is perforated by aqueous pores and dictates the specific tightness characteristics of different epithelia, ranging from almost complete sealing (in the bladder) or forming paracellular pores for specific ions in specific segments of renal tubules. TJs also play a crucial role in the organisation of the transcellular pathway, since they establish the polarity of the epithelial plasma membrane, forming an intramembrane diffusion barrier which restricts the apical-basolateral diffusion of membrane components [1]. This polarization allows ion channels, pumps and enzymes to be differently distributed in apical and basolateral membrane domains establishing the vectorial nature of transepithelial transport in an epithelium [2]. TJs are comprised of three major groups of transmembrane proteins occludin, claudins and junctional adhesion molecules, and a large group of cytoplasmic proteins that form large macromolecular complexes (for full review see [4]). The transmembrane proteins form the paracellular and intramembrane diffusion barriers.

The relative abundance of different claudins that are located in the TJ at any time determine the ion and size selectivity of the paracellular diffusion pathway in that tissue. The studies on the role and distribution of different claudins along the nephron and in renal function has been reviewed in detail by Balkovetz [5]. These studies suggest that the expression of specific claudins along different nephron segments plays a key role in the specific physiological properties of the different nephron segments and leads to the hypothesis that physiological regulation of TJs in the kidney can be controlled in part by modulation of the composition of individual claudin isoforms. In a more recent publication, Balkovetz also summarises the evidence that alterations in claudins in specific nephron segments can be associated with specific kidney diseases including familial hypomagnesaemia and also in a genetic form of human hypertension [6]. Therefore, the regulation of claudins in TJs and the signalling process involved has become a major topic of interest in epithelial cells including renal epithelial cells. The cytoplasmic members of the TJs include a range of adaptor molecules such as the zonula occludens (ZO) proteins, other PDZ-domain-containing proteins and other regulatory components [7]. The adaptor proteins are thought to act as intracellular organising centers to transduce signals between the transmembrane proteins and cytosolic signalling components such as GTPases and protein kinases. Several characterised $\mathrm{TJ}$ adaptor molecules interact with the actin cytoskeleton, and signalling to the actin cytoskeleton is thought to play a critical role in regulating TJ function and barrier permeability [7]. TJs are subject to physiologic regulation and undergo dynamic modulation by agents as diverse as cytokines, calcium, phorbol esters, adenosine triphosphate (ATP), bacterial toxins, and fatty acids. The crosstalk of tight junction components with signalling pathways has been reviewed by Gonzalez-Mariscal et al. [8]. In this article we will review work from own laboratory and others providing evidence that immunosuppressive drugs alter kidney barrier function. The signalling mechanisms involved in altering the TJs and specifically the claudins will also be discussed.

Immunosuppressive drugs are used extensively in organ transplantation and autoimmune disease. The most sensitive indicator of epithelial barrier function in vitro is transepithelial electrical resistance (TEER), which provides a measurement of the degree to which ions can traverse the cell layer [9]. TEER measurement relies on simplified equivalent circuit models where the epithelium is considered as a parallel circuit consisting of two resistive arms - the paracellular and transcellular pathways. The paracellular arm consists of a TJ resistance in series with the lateral intercellular space. The transcellular arm of the circuit consists of an apical membrane resistance in series with a basolateral membrane resistance. Alterations in TEER are generally used as an index of TJ permeability since TJs are rate limiting to paracellular solute movement [9]. It is generally accepted that TEER measurements in relatively low resistance epithelia largely reflects the permeability of the paracellular pathway $[10,11]$. Due to the plasticity and adaptability of TJs, they exert the greatest influence over paracellular permeability of the epithelial barrier, allowing for sensitive modulation of barrier permeability to ions and small molecules.

\section{CYCLOSPORINE A AND SIROLIMUS (RAPAMY- CIN): CLINICAL STUDIES}

Cyclosporine A (CsA) is a calcineurin inhibitor (CNI) which has revolutionized organ transplantation and remains the cornerstone of immunosuppressive therapies to prevent allograft rejection following solid organ transplantation [12]. CsA is also extremely useful in treating autoimmune diseases including severe rheumatoid arthritis and severe psoriasis. The introduction of CsA led to a dramatic reduction in the incidence of acute allograft rejection and an improvement in short-term kidney transplant survival [12]. However CsA has had much less impact on long-term graft survival due to nephrotoxic side effects in patients which ultimately lead to the development of chronic allograft nephropathy (CAN). CAN remains the primary cause of kidney transplant failure [13]. The immunosuppressive actions of CsA are believed to be mediated through prevention of T-cell activation by inhibition of calcineurin, thereby preventing the phosphorylation of NFAT family members responsible for the transcriptional activation of the interleukin-2 and interleukin4 genes [14]. While the underlying cause of CAN is undefined, its development is associated with a number of pathophysiological features such as glomerular and vascular alterations, abnormalities in tubular function and hemodynamic changes (including hypertension). Renal biopsies reveal arteriolar hyalinosis, focal interstitial fibrosis, tubular atrophy, and glomerulosclerosis with focal atrophy [15-17]. A number of mechanisms are proposed to contribute to the observed pathophysiology including systemic, and renal hemodynamic changes, and direct toxic effects of CsA on tubular epithelial cells [18]. Strategies implemented to limit CsA exposure in patients include avoidance, minimisation, and withdrawal [19]. CsA avoidance is associated with in- 
creased rates of acute rejection and these protocols have largely been discontinued [20]. CsA reduction is associated with a modest improvement in renal function, but the development of CAN persists if the CsA exposure continues [20]. The most widely used CsA withdrawal strategies are replacement with, and co-administration with sirolimus (SRL).

SRL is an inhibitor of mammalian target of rapamycin (mTOR) [21]. mTOR phosphorylates a variety of cell cycle intermediates and catalyzes processes necessary for gene transcription and protein translation. SRL inhibits the p70S6 kinase necessary for the synthesis of endoplasmic reticulum structural proteins [22]. Inhibition of mTOR also affects critical events later in the cell cycle including $\mathrm{p} 27^{\mathrm{kip} 1}$ degradation [23] and release of elongation initiation factor (eIF) 4A, which facilitates protein synthesis by ribosomes [24]. Together, these effects result in inhibition of IL-2mediated signal transduction pathways and results in inhibition of proliferation and clonal expansion of $\mathrm{T}$ and $\mathrm{B}$ cells stimulated by cytokines or growth factors. This mechanism of action complements that of CNIs.

The two major strategies for SRL therapy in clinical organ transplantation are de novo use of SRL in combination with reduced amounts of CsA, or complete conversion from a CNI-based protocol to SRL in cases where well-recognized adverse effects of CNIs (such as impaired renal function) are prevalent [20]. Although the hemodynamic and renal side effects of SRL are significantly less, compared to those of CsA, other serious adverse effects such as lipid abnormalities and thrombocytopenia have been reported [25]. Furthermore, there is increasing evidence of enhanced nephrotoxicity and proteinuria in clinical studies where CsA and SRL are used in combination [26-28]. These findings have prompted much discussion and long-term follow-up studies in large cohorts are required to determine whether replacement of CsA with SRL provides any significant improvement in patient and graft survival [20].

\section{CSA AND SRL: IN VIVO EXPERIMENTAL STUDIES}

The apparent contradictory nature of clinical findings regarding CsA and SRL combination therapies has prompted a significant number of in vivo and in vitro experimental studies. By and large, these studies have attempted to delineate the effects of CsA and SRL, individually or in combination. Since CsA was developed much earlier than SRL, it is understandable that many more in vivo studies have been performed using CsA. A range of rodent models of CsA nephrotoxicity have been developed. Earlier work from our laboratory demonstrated altered magnesium homeostasis involving renal magnesium wasting in a rat model of CsA nephrotoxicity [29]. More recently, we have developed a mouse model of CsA nephrotoxicity and used proteomic techniques to identify possible novel biomarkers [30]. However, for this present discussion, the focus will be on studies that examined potential effects on renal transport. Kim et al. [31] demonstrated that CsA treatment significantly reduced proteinuria and the diminished glomerular $\mathrm{ZO}-1$ expression in puromycin aminonucleoside induced nephrosis in rats. However, in another model of protein overload involving repeated injections of bovine serum albumin, Cai et al. [32] demonstrated that SRL aggravated proteinuria.
A number of studies have investigated alterations in epithelial transporters with CsA treatment. Esteva-Font $e$ t al. [33] demonstrated an increase in the $\mathrm{Na}-\mathrm{K}-2 \mathrm{Cl}$ ( $\mathrm{NKCC} 2$ ) cotransporter in the loop of Henle in rats treated with CsA $(25 \mathrm{mg} / \mathrm{kg} /$ day IP for 7 days). Lee et al. [34] demonstrated a decrease in $\mathrm{Na}-\mathrm{Cl}$ cotransporter in renal cortex of rats treated with CsA and also demonstrated that occludin was also increased in rat kidneys exposed to CsA. Our earlier finding of magnesium wasting in CsA toxicity [29] may be provided a mechanistic basis in a study of Chang et al. [35] who demonstrated in an immortalized cultured thick ascending limb cell line that CsA significantly reduced parcellin-1 (claudin 16). Paracellin-1 is a tight junction protein which regulates the paracellular transport of magnesium in the tick ascending limb.

\section{EFFECTS OF CSA AND SRL ON TUBULAR EPITHELIAL CELLS}

Since the renal tubular interstitium is a primary site of toxicity during CAN, there has been particular focus on tubular epithelial cells of the nephron (primarily proximal and distal tubule cells) in investigations of CsA and SRL effects on the kidney. Tubular epithelial cells play a central role in the initiation and development of CAN via a variety of mechanisms including secretion of profibrotic chemokines and growth factors [36, 37], epithelial-to-mesenchymal transition, extracellular matrix accumulation and renal fibrosis [38]. A wide range of studies have examined the direct effects of CNIs on tubular epithelial cells in vitro primarily focusing on models of the proximal and distal tubules, however some data relating to the loop of Henle is also available. However, one has to carefully consider the experimental conditions in which studies on the renal epithelial cells has been carried out in vitro. For example, many studies have been carried out on cells under sub-confluent conditions when the transport properties of the cells have not fully established. None the less, these studies do show clearly that CsA and SRL have direct effects on kidney epithelial cells.

CsA has been shown to increase apoptosis in tubular [39] and interstitial cells by inducing tubular atrophy [40], inflammatory mediators, enhanced immunogenicity [41, 42] and epithelial-mesenchymal transition in tubular epithelial cells $[43,44]$. In the porcine proximal tubule cell line LLCPK1, CsA caused increased apoptosis, cell cycle arrest and decreased cell proliferation [39, 45]. CsA has also been shown to increase levels of the tumor suppressor p53 in LLC-PK1 cells with associated cell cycle arrest [46]. In the human proximal tubular cell line, HK-2, CsA exposure results in stress-induced senescence in both HK-2 cells and primary proximal tubular cells, characterized by a reduced proliferation, decreased DNA synthesis and p53 activation [47]. In sub-confluent HK-2 cells, CsA has been shown to induce epithelial mesenchymal transition (EMT) and promote fibrotic effects [43, 44]. In the distal tubular MadinDarby canine kidney (MDCK) cell line, CsA induced cell cycle arrest, apoptosis and significant increases in lactate dehydrogenase activity [48].

By comparison, the direct effects of SRL on tubular epithelial cells have been less well studied. SRL has been shown to inhibit TGF- $\beta 1$-induced EMT in proximal tubular cells suggesting an anti-fibrotic effect [49]. Pallet et al, [50] 
demonstrated that SRL dramatically decreased the proliferative response of tubular epithelial cells to mitogenic stimuli at clinically relevant concentrations. In 2008, the same group reported on a toxicogenomic study of CsA and SRL effects on the tubular epithelial cell gene transcription profile [51]. In summary, this study demonstrated that SRL elicited dramatically different effects on the gene expression profile, dependent on whether it was administered alone or in combination with CsA. On one hand, CsA and $\mathrm{CsA}+\mathrm{SRL}$ induced similar transcriptional changes, inducing profibrotic genes and affecting genes concerned with ion transport or ligand receptor interaction. In contrast, SRL, when administered alone, was reported to regulate genes involved in transcriptional activity, cell death and cellular metabolism.

\section{EFFECTS OF CYCLOSPORINE A AND SIROLIMUS ON TUBULAR EPITHELIAL CELL BARRIER FUNCTION}

While these studies provide clear evidence of direct effects of CsA and SRL on tubular epithelial cells, they did not directly examine the effects of CsA and SRL on tubular epithelial cell transport and barrier function. In a series of studies from our group, the effects of CsA on barrier function in MDCK II cells were investigated [52-54]. It was demonstrated that sub-cytotoxic concentrations of CsA decreased the paracellular permeability of MDCK II cell monolayers, detected as an increase in TEER [53]. Inhibitor studies demonstrated that activation of the extracellular signalregulated kinase 1/2 (ERK1/2) mitogen-activated protein kinase (MAPK) cascade was central to the CsA-induced increase in TEER. In follow up studies, we demonstrated that alterations in claudin-1 and zonula occludens 2 (ZO-2) expression were observed following CsA. [52]. We also demonstrated that induction of TGF- $\beta 1$ and the TGF- $\beta$ type II receptor were involved in the CsA-mediated increase in barrier function and TEER. We extended the findings with the MDCK cells into a model of the proximal tubule using the LLC-PK1 cell line [54]. In these studies with LLC-PK1 cells, CsA exposure induced a significant increase in TEER and this effect coincided with alterations in the expression of TJ proteins, claudins- $1,-2$ and -4 with claudins -1 and -4 being upregulated and claudin-2 being downregulated. TGF$\beta 1$ induction and ERK1/2 signaling were also central to the CsA-mediated alterations in proximal tubular epithelial cell barrier function. Furthermore, the effects of SRL alone and in combination with CsA were also examined in these studies. A subcytotoxic concentration of SRL was also found to significantly decrease paracellular permeability of LLC-PK1 cell monolayers. Surprisingly, cotreatment of cells with SRL and CsA was found to further decrease paracellular permeability revealing a significant synergistic effect between the two drugs. The effects of CsA and SRL alone and in combination on the TEER are shown in Fig. (1). This synergism appeared to be underpinned by co-regulation of TGF- $\beta 1$ production resulting in downstream ERK1/2 signalling, and alterations in claudin-1, -2 and -4 regulation. Additional unpublished data from our laboratory indicates that TGF- $\beta 1$ induced ERK $1 / 2$ signalling is critical for the effects of CsA and/or SRL on barrier function and that SMAD signalling is of lesser significance.

A recent study by Lee et al. [34] also observed modulation of TEER by CsA, but in MDCK I cells which exhibit a very high basal TEER. They reported that CsA at a concentration of $83 \mathrm{nM}$ decreased TEER but, in agreement with our previous findings in MDCKII cells, observed that CsA at a higher concentration of $0.4 \mu \mathrm{M}$ induced a significant increase in TEER. Alterations in ZO-1 and occludin 1 were observed but no changes in claudin- 1 or -4 were reported by the investigators. Any possible mechanistic differences are likely due

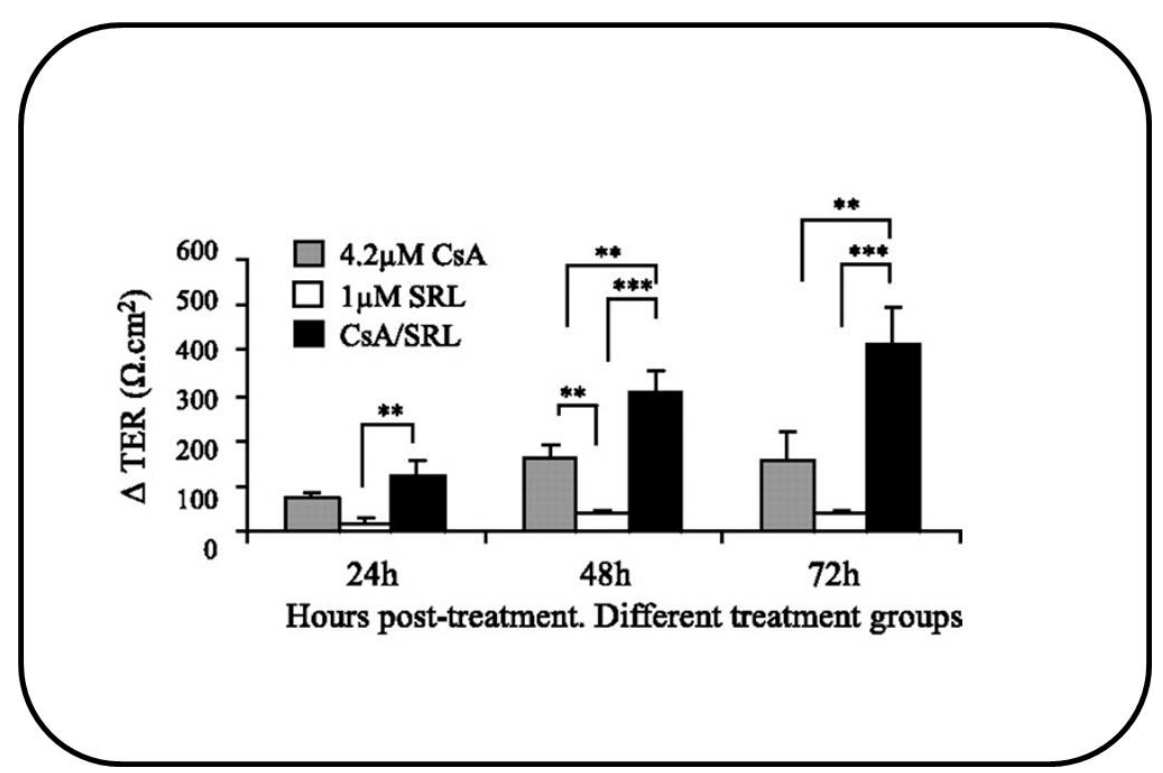

Fig. (1). Effects of CsA and SRL on proximal tubular epithelial cell barrier function. Adapted from Martin-Martin N et al. Am J Physiol Renal Physiol 2010; 298: F672-F682 (C2010 by American Physiological Society). Cyclosporine A (CsA), sirolimus (SRL), and CsA/SRL cotreatment increased transepithelial electrical resistance (TEER). LLC-PK1 cells were grown on 24-Costar Transwell filters and treated with either vehicle control $(\mathrm{EtOH}), 4.2 \mu \mathrm{M} \mathrm{CsA}, 1 \mu \mathrm{M}$ SRL, or $4.2 \mu \mathrm{M}$ CsA $+1 \mu \mathrm{M}$ SRL for periods up to $72 \mathrm{~h}$. Results are expressed as the change in TEER $(\triangle \mathrm{TER})$ compared with time-matched control filters and are given as means \pm SE of 3 independent experiments performed in triplicate. $\triangle \mathrm{TER}$ is expressed as $\Omega \cdot \mathrm{cm} 2$. 
to physiological differences between the MDCK I and MDCK II cell lines. For example, MDCK II cells exhibit a lower basal resistance than MDCK I cells $\left(\sim 100 \Omega / \mathrm{cm}^{2}\right.$ versus $4,000-10,000 \Omega / \mathrm{cm}^{2}$ ). This difference in basal TEER is likely mediated by the fact that MDCK I cells do not express claudin-2 (a major pore-forming claudin), where as MDCK II cells do. However, it is also possible that CsA at a concentration of $83 \mathrm{nM}$ may have induced apoptosis as we previously provided evidence that $\mathrm{CsA}$ in the nano molar range can induce apoptosis [39].

To our knowledge, there has been only one other study apart from our own [54] discussed above which reported effects of SRL on TEER in kidney cells [55]. Zhang et al. in a study investigating the role of AMP-activated protein kinase in regulation of the assembly of epithelial tight junctions in MDCK I cells reported that SRL at a concentration of 20nM had a beneficial effect on TEER and partially rescued delayed ZO-1 localization caused by dominant negative AMPK expression [55]. SRL has been shown to inhibit receptor mediated endocytosis of albumin in human proximal tubular cells in an angiotensin II-dependent manner [56]. With respect to the distal tubule, SRL has been shown to inhibit GLUT12 translocation in MDCK cells [57]. It remains to be established if CsA, SRL or indeed CsA and SRL in combination modulate the expression and/or localisation of a range of other transcellular transporters and of other TJ proteins including additional claudins in proximal and distal tubular epithelial cells.

The effects of CsA and/or SRL on kidney cells in vitro may appear contradictory. On the one hand observed effects include cell cycle arrest, apoptosis, ER stress and EMT, and on the other increased barrier function and increased TEER. However, it is likely that these different effects are mainly due to the specific conditions under which the experiments were carried out. We have summarized these reports and effects observed in Table 1. This clearly shows that the effects induced by CsA and/or SRL such as cell cycle arrest, apoptosis and EMT have been reported in studies with subconfluent cells grown on plastic. On the other hand, the studies on barrier function and TEER have, of course, been carried out on confluent cells grown on filters. The hypothesis would be that these latter conditions may be closer to the in vivo situation with intact epithelial barrier function. However during situations of injury and repair, sub-confluency of cells and cell division may also be very relevant.

\section{CONCLUSIONS}

In conclusion, evidence presented indicates that CsA, SRL, and CsA/SRL in combination significantly alter renal transport including the paracellular permeability of renal tubular epithelial cells, resulting in enhanced barrier function. While the synergism observed between CsA and SRL is somewhat surprising considering their indicated diverse mechanisms of immunosuppressive action. The results from our laboratory identified TGF- $\beta 1$ and ERK MAPK signalling as potent regulators of paracellular permeability in both proximal and distal tubular cells after treatment with CsA, SRL and CsA/SRL. The possible mechanisms are presented diagrammatically in Fig. (2). The fact that the CsA and SRL effects on TEER and barrier function are mediated, at least in part by TGF- $\beta 1$ also suggests that the effects may be observed in other epithelia. TGF- $\beta 1$ treatment has been shown to enhance the barrier function of intestinal epithelial monolayers and promoted intestinal epithelial restitution [58, 59]. Conversely, TGF- $\beta 1$ inhibited glucocorticoid-stimulated tight junction formation and decreased TEER in murine mammary epithelial cells [60]. Together, these observations

Table 1. Effects of CsA and SRL on Tubular Epithelial Cells In Vitro

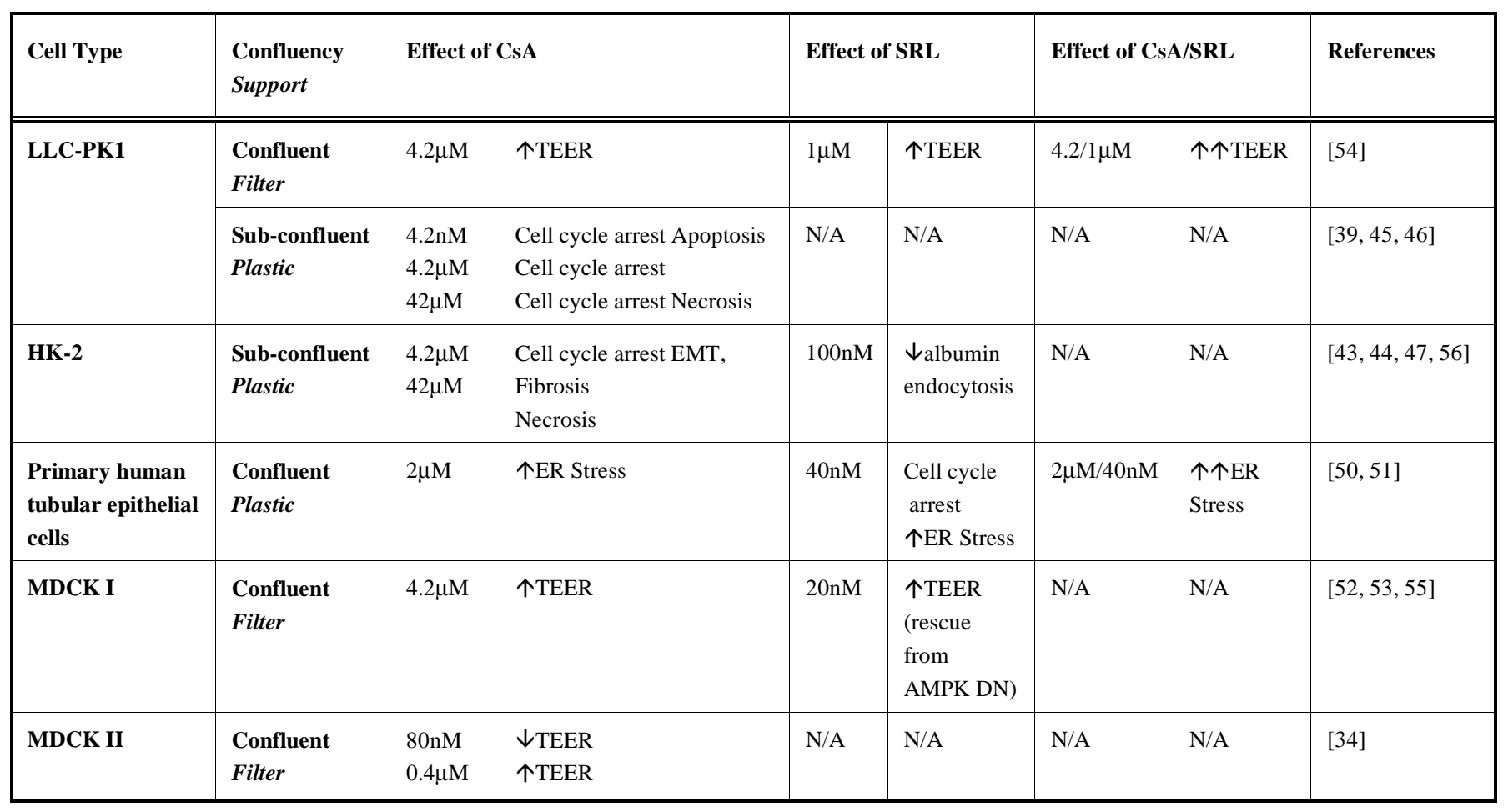




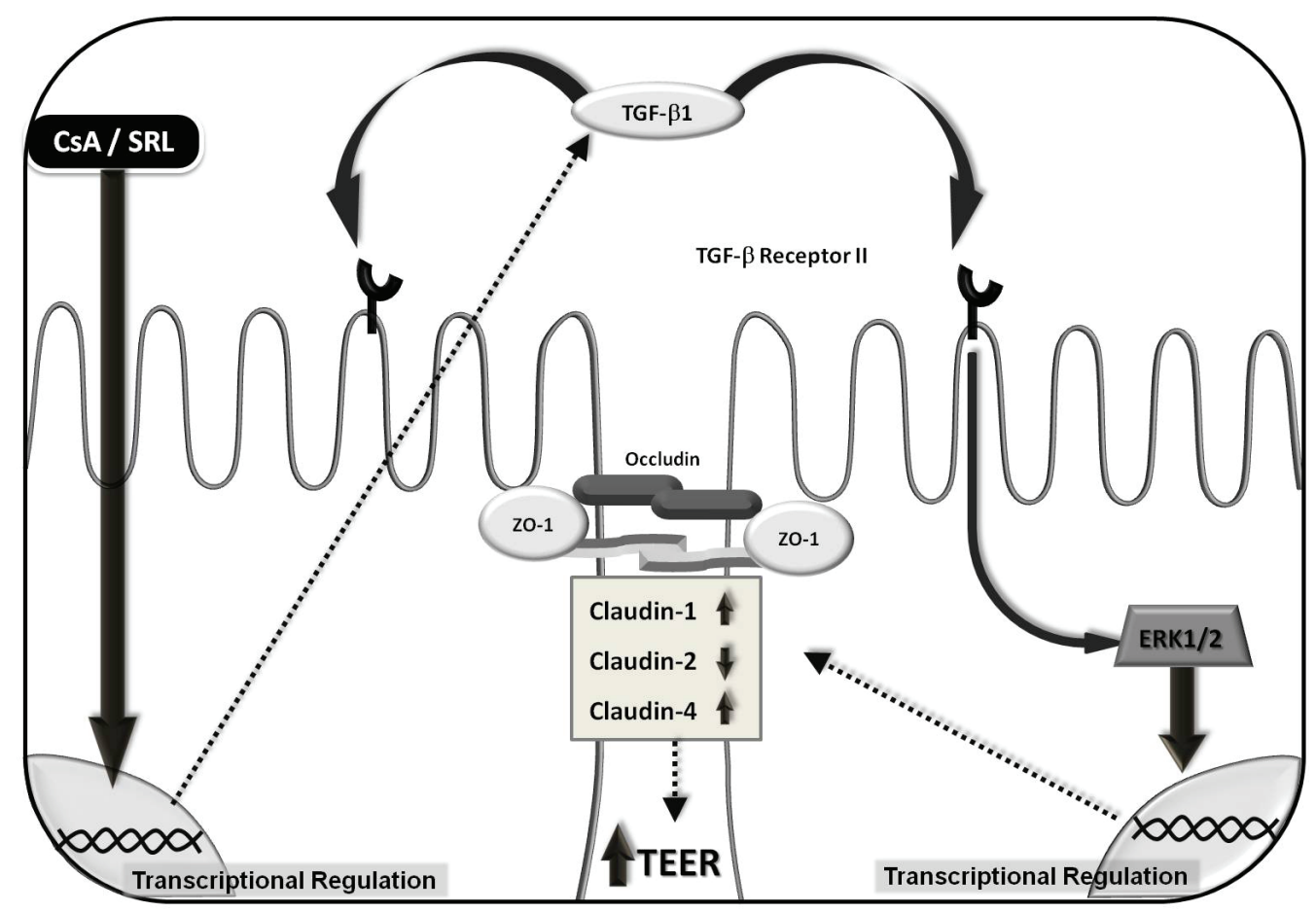

Fig. (2). Proposed mechanism of immunosuppressant modulation of tubular epithelial barrier function. CsA and SRL-induced changes in TEER are mediated by alterations in expression and localisation of claudins- $1,-2$ and -4 . These effects are dependent on TGF- $\beta 1$ and ERK1/2 signalling.

suggest that TGF- $\beta 1$ elicits diverse effects on epithelial barrier function in different tissues.

The effects of CsA and SRL on kidney epithelial cell barrier function may provide explanation of some of the renal transport defects and nephrotoxicity observed in patients treated with CsA and SRL. However, if similar effects occur systemically in vivo with these two immunosuppressive drugs, the enhancement of barrier function in areas of the body may decrease luminal antigen presentation, decreasing immune activation and support the immunosuppressive action of these drugs. The mechanisms underlying these effects may differ subtly between cell models but the overarching importance of these observations and their potential clinical implications highlight the need for further characterization of the molecular mechanisms underlying these drug-induced alterations in barrier function.

\section{CONFLICT OF INTEREST}

None declared.

\section{ACKNOWLEDGEMENTS}

The authors work in this area was funded by the Health Research Board of Ireland and the EU 7th Framework grant "SysKid", HEALTH-F2-2009-241544. Dr. Craig Slattery is a Government of Ireland Research Fellow funded by the Irish Research Council for Science, Engineering and Technology. UCD Conway Institute is funded by the programme for research in third level institutions (PRTLI) administered by the Higher Education Authority.

\section{REFERENCES}

[1] Gumbiner BM. Breaking through the tight junction barrier. J Cell Biol 1993; 123: 1631-3.
[2] Schneeberger EE, Lynch RD. Structure, function, and regulation of cellular tight junctions. Am J Physiol 1992; 262(6 Pt 1): L647-61.

[3] Farquhar MG, Palade GE. Junctional complexes in various epithelia. J Cell Biol 1996; 17: 375-412.

[4] Van Itallie CM, Anderson JM. Claudins and epithelial paracellular transport. Annu Rev Physiol 2006; 68: 403-29.

[5] Balkovetz DF. Claudins at the gate: determinants of renal epithelial tight junction paracellular permeability. Am J Physiol Renal Physiol 2006; 290: F572-9.

[6] Balkovetz DF. Tight junction claudins and the kidney in sickness and in health. Biochim Biophys Acta 2009; 1788: 858-63.

[7] Wittchen ES, Haskins J, Stevenson BR. Protein interactions at the tight junction. Actin has multiple binding partners, and $\mathrm{ZO}-1$ forms independent complexes with ZO-2 and ZO-3. J Biol Chem 1999; 274: 35179-85.

[8] Gonzalez-Mariscal L, Tapia R, Chamorro D. Crosstalk of tight junction components with signaling pathways. Biochim Biophys Acta 2008; 1778: 729-56.

[9] Duff T, S Carter, G Feldman, et al. Transepithelial resistance and inulin permeability as endpoints in in vitro nephrotoxicity testing. Altern Lab Aim 2002; 30 Suppl 2: 53-9.

[10] Gonzalez-Mariscal L, Chavez de Ramirez B, Lazaro A, Cereijido M, Carters S, Feldman C. Establishment of tight junctions between cells from different animal species and different sealing capacities. J Membr Biol 1989; 107: 43-56.

[11] Reuss L, Simon B, Cotton CU. Pseudo-streaming potentials in Necturus gallbladder epithelium. II. The mechanism is a junctional diffusion potential. J Gen Physiol 1992; 99: 317-38.

[12] Halloran PF. Immunosuppressive drugs for kidney transplantation. N Engl J Med 2004; 351: 2715-29.

[13] Nankivell BJ, Borrows RJ, Fung CL, et al. The natural history of chronic allograft nephropathy. N Engl J Med 2003; 349: 2326-33.

[14] Fruman DA, Klee CB, Bierer BE, Burakoff SJ. Calcineurin phosphatase activity in T lymphocytes is inhibited by FK 506 and cyclosporin A. Proc Natl Acad Sci USA 1992; 89: 3686-90.

[15] Mihatsch MJ, Ryffel B, Hermle M, et al. Morphology of cyclosporine nephrotoxicity in the rat. Clin Nephrol 1986; 25 Suppl 1: S2-8.

[16] Mihatsch MJ, Thiel G, Ryffel B. Morphologic diagnosis of cyclosporine nephrotoxicity. Semin Diagn Pathol 1988; 5: 104-21. 
[17] Whiting PH, Thomson AW, Simpson JG, Cyclosporine. toxicity, metabolism, and drug interactions--implications from animal studies. Transplant Proc 1985. 17(4 Suppl 1): 134-44.

[18] Campistol JM, Sacks SH. Mechanisms of nephrotoxicity. Transplantation 2000; 69(12 Suppl): SS5-10.

[19] Reis FN. The unsolved cyclosporine-induced kidney injury: is paricalcitol a feasible new renoprotective option? Kidney Int 2010; 77: 1055-7.

[20] Flechner SM, Kobashigawa J, Klintmalm G. Calcineurin inhibitorsparing regimens in solid organ transplantation: focus on improving renal function and nephrotoxicity. Clin Transplant 2008; 22: 1-15.

[21] Sabatini DM, Erdjument-Bromage H, Lui M, et al. RAFT1: a mammalian protein that binds to FKBP12 in a rapamycin-dependent fashion and is homologous to yeast TORs. Cell 1994; 78: 35-43.

[22] Kuo CJ, Chung J, Fiorentino DF, et al. Rapamycin selectively inhibits interleukin-2 activation of p70 S6 kinase. Nature 1992; 358: 70-3.

[23] Luo Y, Marx SO, Kiyokawa H, et al. Rapamycin resistance tied to defective regulation of p27Kip1. Mol Cell Biol 1996; 16: 6744-51.

[24] Hara K, Yonezawa K, Kozlowski MT, et al. Regulation of eIF-4E BP1 phosphorylation by mTOR. J Biol Chem 1997; 272: 26457-63.

[25] Hong JC, Kahan BD. Sirolimus-induced thrombocytopenia and leukopenia in renal transplant recipients: risk factors, incidence, progression, and management. Transplantation 2000; 69: 2085-90.

[26] Kahan BD, Napoli KL, Kelly PA, et al. Therapeutic drug monitoring of sirolimus: correlations with efficacy and toxicity. Clin Transplant 2000; 14: 97-109.

[27] MacDonald AS, worldwide A. phase III, randomized, controlled, safety and efficacy study of a sirolimus/cyclosporine regimen for prevention of acute rejection in recipients of primary mismatched renal allografts. Transplantation 2001; 71: 271-80.

[28] MacDonald AS. Rapamycin in combination with cyclosporine or tacrolimus in liver, pancreas, and kidney transplantation. Transplant Proc 2003; 35: 201S-208S.

[29] Clarke H, Ryan MP. Cyclosporine A-induced alterations in magnesium homeostasis in the rat. Life Sci 1999; 64: 1295-306.

[30] O'Connell S, Slattery SC, Ryan MP, McMorrow T. Identification of novel indicators of cyclosporine A nephrotoxicity in a CD-1 mouse model. Toxicol Appl Pharmacol 2011; 252: 201-10.

[31] Kim BS, Park HC, Kang SW, et al. Impact of cyclosporin on podocyte ZO-1 expression in puromycin aminonucleoside nephrosis rats. Yonsei Med J 2005; 46: 141-8.

[32] Cai Y, Chen Y, Zheng S, et al. Sirolimus damages podocytes in rats with protein overload nephropathy. J Nephrol 2011; 24(3): 307-12.

[33] Esteva-Font C, Ars E, Guillen-Gomez E, et al. Ciclosporin-induced hypertension is associated with increased sodium transporter of the loop of Henle (NKCC2). Nephrol Dial Transplant 2007; 22: 2810-6.

[34] Lee CH, Kim S, Kang CM, et al. Altered expression of tight junction proteins in cyclosporine nephrotoxicity. Am J Nephrol 2011; 33: 7-16.

[35] Chang CT, Hung CC, Tian YC, et al. Ciclosporin reduces paracellin-1 expression and magnesium transport in thick ascending limb cells. Nephrol Dial Transplant 2007; 22: 1033-40.

[36] Eddy AA, Neilson EG. Chronic kidney disease progression. J Am Soc Nephrol 2006; 17: 2964-6.

[37] Iwano M, Plieth D, Danoff TM, et al. Evidence that fibroblasts derive from epithelium during tissue fibrosis. J Clin Invest 2002; 110: 341-50.

[38] Zeisberg M, Maeshima Y, Mosterman B, Kalluri R, Fibrosis R. Extracellular matrix microenvironment regulates migratory behavior of activated tubular epithelial cells. Am J Pathol 2002; 160: 2001-8.

[39] Healy E, Dempsey M, Lally C, Ryan MP. Apoptosis and necrosis: mechanisms of cell death induced by cyclosporine $\mathrm{A}$ in a renal proximal tubular cell line. Kidney Int 1998; 54: 1955-66.

[40] Shihab FS, Andoh TF, Tanner AM, et al. Role of transforming growth factor-beta 1 in experimental chronic cyclosporine nephropathy. Kidney Int 1996; 49: 1141-51.
[41] Burdmann EA, Andoh TF, Yu L, Bennett WM. Cyclosporine nephrotoxicity. Semin Nephrol 2003; 23: 465-76.

[42] Burdmann EA, Young B, Andoh TF, et al. Mechanisms of cyclosporine-induced interstitial fibrosis. Transplant Proc 1994; 26 : 2588-9.

[43] McMorrow T, Gaffney MM, Slattery C, et al. Cyclosporine A induced epithelial-mesenchymal transition in human renal proximal tubular epithelial cells. Nephrol Dial Transplant 2005; 20: 2215-25.

[44] Slattery C, Campbell E, McMorrow T, Ryan MP. Cyclosporine Ainduced renal fibrosis: a role for epithelial-mesenchymal transition. Am J Pathol 2005; 167: 395-407.

[45] Becker GM, Gandolfi AJ, Nagle RB. Effects of cyclosporin A on a kidney epithelial cell line (LLC-PK1). Res Commun Chem Pathol Pharmacol 1987; 56: 277-80.

[46] Lally C, Healy E, Ryan MP. Cyclosporine A-induced cell cycle arrest and cell death in renal epithelial cells. Kidney Int 1999; 56: 1254-7.

[47] Jennings P, Koppelstaetter C, Aydin S, et al. Cyclosporine A induces senescence in renal tubular epithelial cells. Am J Physiol Renal Physiol 2007; 293: F831-8.

[48] Rezzani R, Angoscini P, Borsani E, et al. Cyclosporine A-induced toxicity in two renal cell culture models (LLC-PK1 and MDCK). Histochem J 2002; 34: 27-33.

[49] Copeland JW, Beaumont BW, Merrilees MJ, Pilmore HL. Epithelial-to-mesenchymal transition of human proximal tubular epithelial cells: effects of rapamycin, mycophenolate, cyclosporin, azathioprine, and methylprednisolone. Transplantation 2007; 83: 809-14.

[50] Pallet N, Thervet E, Le Corre D, et al. Rapamycin inhibits human renal epithelial cell proliferation: effect on cyclin D3 mRNA expression and stability. Kidney Int 2005; 67: 2422-33.

[51] Pallet N, Rabant M, Xu-Dubois YC, et al. Response of human renal tubular cells to cyclosporine and sirolimus: a toxicogenomic study. Toxicol Appl Pharmacol 2008. 229: 184-96.

[52] Feldman G, Kiely B, Martin N, et al. Role for TGF-beta in cyclosporine-induced modulation of renal epithelial barrier function. $\mathrm{J}$ Am Soc Nephrol 2007; 18: 1662-71.

[53] Kiely B, Feldman G, Ryan MP. Modulation of renal epithelial barrier function by mitogen-activated protein kinases (MAPKs): mechanism of cyclosporine A-induced increase in transepithelial resistance. Kidney Int 2003; 63: 908-16

[54] Martin-Martin N, Ryan G, McMorrow T, Ryan MP. Sirolimus and cyclosporine A alter barrier function in renal proximal tubular cells through stimulation of ERK1/2 signaling and claudin-1 expression. Am J Physiol Renal Physiol 2010; 298: F672-82.

[55] Zhang L, Li J, Young LH, Caplan MJ. AMP-activated protein kinase regulates the assembly of epithelial tight junctions. Proc Natl Acad Sci USA 2006; 103: 17272-7.

[56] Oroszlan M, Bieri M, Ligeti N, et al. Sirolimus and everolimus reduce albumin endocytosis in proximal tubule cells via an angiotensin II-dependent pathway. Transpl Immunol 2010; 23: 125-32.

[57] Wilson-O'Brien AL, Dehaan CL, Rogers S. Mitogen-stimulated and rapamycin-sensitive glucose transporter 12 targeting and functional glucose transport in renal epithelial cells. Endocrinology 2008. 149: 917-24.

[58] Dignass AU, Podolsky DK. Cytokine modulation of intestinal epithelial cell restitution: central role of transforming growth factor beta. Gastroenterology 1993; 105: 1323-32.

[59] Planchon S, Fiocchi C, Takafuji V, Roche JK. Transforming growth factor-betal preserves epithelial barrier function: identification of receptors, biochemical intermediates, and cytokine antagonists. J Cell Physiol 1999; 181: 55-66.

[60] Guan Y, Woo PL, Rubenstein NM, Firestone GL. Transforming growth factor-alpha abrogates the glucocorticoid stimulation of tight junction formation and reverses the steroid-induced downregulation of fascin in rat mammary epithelial tumor cells by a Rasdependent pathway. Exp Cell Res 2002; 27: 1-11. 